\title{
Drug delivery systems for potential treatment of intracellular bacterial infections
}

\section{Edurne Imbuluzqueta ${ }^{1}$, Carlos Gamazo², Javier Ariza ${ }^{3}$, Maria J. Blanco-Prieto ${ }^{1}$}

${ }^{1}$ Department of Pharmacy and Pharmaceutical Technology, University of Navarra, 31080 Pamplona, Spain, ${ }^{2}$ Department of Microbiology and Parasitology, University of Navarra, 31080 Pamplona, Spain, ${ }^{3}$ Infectious Disease Service, Hospital Universitario de Bellvitge, IDIBELL, L'Hospitalet de Llobregat, Barcelona, Spain

\section{TABLE OF CONTENTS}

\section{Abstract}

2. Introduction

3. Lifestyles of intracellular bacterial pathogens

3.1. Lysis of the intracellular vacuole and escape to the cytosol

3.2. Arrest of phagosome maturation

3.3. Segregation from the endocytic route

3.4. Life within acidic phagosomes

4. Antibiotic treatment of intracellular bacterial infections

5. Antibiotic encapsulation in drug delivery systems

5.1. Liposomes

5.1.1. Uptake of liposomes by mononuclear phagocytic system cells

5.1.2. Intracellular destination of $\mathrm{pH}$-sensitive liposomes

5.1.3. In vitro efficacy of liposome-assisted antibiotic treatment

5.2. Polymeric micro- and nanoparticles

5.2.1. Endocytosis and intracellular fate of particles used as drug delivery systems

5.2.2. Synergy of polymeric particles with cellular bactericidal mechanisms

5.2.3. In vitro efficacy of polymeric particles for antibiotic treatment

6. Treatment of experimental infections mediated by drug delivery systems

6.1. Tuberculosis

6.2. Brucellosis

6.3. Salmonellosis

6.4. Listeriosis

7. Concluding remarks and perspectives

8. Acknowledgment

9. References

\section{ABSTRACT}

Despite the advent of a considerable number of new antibiotics, treatment of intracellular pathogens still represents a major pharmaceutical challenge. The antibiotic concentration in those specialized niches are often subtherapeutic, for which high doses of antibiotics must often be used. This is not only costly but may also increase localized or systemic side effects. There is therefore an urgent need for materials and methods to enable clinicians to achieve therapeutically effective intracellular concentration of those antibiotics which show good efficiency in vitro. In this setting, the possible use of drug delivery systems (DDS) loaded with antibiotics that exhibit a high in vitro bactericidal activity deserves to be considered. Entrapping or encapsulating the drug within a delivery system provides a greater control of the pharmacokinetic behavior of the active molecule. This more efficient use of antibiotics may diminish their drawbacks and provide the basis for shortening the current time required by classical treatments. This review will focus on the role of DDS as a potential tool against intracellular bacteria.

\section{INTRODUCTION}

The chemotherapy of infections caused by bacteria that reside intracellularly presents a number of unusual challenges. On the one hand, several bacteria have found the way to produce a "silent" infection inside the cells and to escape from their bactericidal mechanisms. Thus, in these situations, cells are not only incompetent to eradicate the intracellular bacteria but also may act as reservoirs and contribute to the spreading of the infection to other cells and organs. Moreover, due to their intracellular location bacteria are protected from the attack of the immune system as well as from the action of the antibiotics present in the extracellular milieu. Thus, although a wide variety of antibiotics have been found to be active against isolated bacteria, disappointing results are obtained against these pathogens in the intracellular environment (1). On the other hand, since antibiotic treatment of intracellular infections is not always successful, long-lasting combined therapeutic regimens are usually needed. However, although combined therapies are more effective than single ones, their fulfilment becomes more difficult for patients and this can lead to a poor patient compliance and abandoning of the antibiotic treatment. Taken together, all these factors may contribute to the occurrence of relapses after treatment. 


\section{DDS against intracellular infections}

In this context, the current review will focus on the potential utility of antibiotic-containing DDS in the treatment of intracellular infections. These DDS can deliver antimicrobial agents and target intracellular sites of infection, thereby helping to increase the therapeutic index of antimicrobials in intracellular niches, while avoiding problems associated with administering high doses of antibiotics systemically.

\section{LIFESTYLES OF INTRACELLULAR BACTERIAL PATHOGENS}

A significant niche to be considered in the area of infectious diseases is the intracellular milieu of the professional phagocytes. Paradoxically, these specialized cells tailored to kill and digest the ingested material are a satisfying habitat for bacterial pathogens such as Listeria, Brucella, Mycobacterium or Salmonella. The strong evolutionary pressure for overcoming the lethal immune system challenge has resulted in a diverse repertoire of microbial strategies to create a safe replicative niche. Intracellular (obligate or facultative) pathogens present different virulence factors that will modulate their intracellular fate (2).

Figure 1 shows the classic action of the phagocyte to kill and degrade the ingested bacteria. The endocytic pathway is used for the uptake of nutrients and recycling of proteins used in the secretory pathway, among other functions. Phagosomes containing ingested bacteria fuse with early endosomes, acquiring markers that confer on phagosomes properties normally assigned to early endosomes, including the ability to fuse with other endocytic organelles $(3,4)$. They fuse sequentially with endosomes of increasing age or maturation level (late endosomes and lysosomes) acquiring new markers and a more acidic environment $(\mathrm{pH}$ 5.5). Changes in the phospholipid composition is also observed during phagosome maturation; while early phagosomes are enriched with phosphatidylcholine, late phagosomes are preferentially enriched with sphingomyelin (5). Altogether, these changes contribute to the formation of the phagolysosome, an acidic compartment displaying not only the harsh and lytic environment needed to kill and degrade the ingested material (4), but also a harsh milieu to maintain the bioactivity of many drugs. Intracellular bacteria have found ways to divert their usual trafficking from early endosomes towards these inhospitable phagolysosomes. Pathogens can thus escape from the endocytic vacuole to the cytoplasm, live within the phagolysosome, or create their own privileged niche (Table 1) (6-22). Regardless of the means by which this is achieved, pathogens can survive and replicate undetected within the protected niche of the host cell. Several pathogens represent paradigmatic models for intracellular survival mechanisms.

\subsection{Lysis of the intracellular vacuole and escape to the cytosol}

Listeria and Shigella are examples of pathogens which can escape from the phagocytic vacuole avoiding exposure to the degradative compartments of the endocytic route, and therefore are finally found at the neutral $\mathrm{pH}$ and nutrient-rich host cytosol. Listeria monocytogenes has the ability to enter and survive into both phagocytic and non-phagocytic cells and it invades the cytosol thanks to a pore-forming hemolysin (listeriolysin $\mathrm{O}$ ) and some phospholipases (a phosphatidylinositol-specific phospholipase $\mathrm{C}$ and a broad-spectrum phospholipase $\mathrm{C}$ ) (23). Listeriolysin $\mathrm{O}$ forms pores in the vacuole membrane allowing the release of the phospholipases which cause the disruption of this Listeria-containing vacuole membrane. Once in the cytosol the bacterium induces the nucleation of host actin filaments and the formation of an actin tail that is able to push the bacterium into the neighboring cells, in which it reinitiates this lifecycle (7). In the case of Shigella flexneri a cytotoxin lyses the membrane of the phagosome and allows it to escape to the cytosol. Like Listeria, Shigella exploits the host actin polymerization machinery and induces the formation of actin-rich comet tails by means of a protein called IcsA (24).

\subsection{Arrest of phagosome maturation}

Other pathogenic bacteria such as Salmonella and Mycobacterium arrest the maturation of the phagosome at specific stages of the phago-lysosomal route and evolve into an intracellular life cycle involving prevention of phagosome-lysosome fusion. As a consequence, the $\mathrm{pH}$ of the final localization of the bacteria is close to neutrality. Salmonella typhimurium transiently acquires early endosome markers but it has developed mechanisms to modulate the redistribution of endosomal and lysosomal markers and it seems that it may modulate the expression of vacuolar ATPases to generate a relatively less acidic phagosomal compartment (25).

Professional phagocytes are the preferred targets of Mycobacterium spp. Soon after its phagocytosis, Mycobacteriumcontaining vacuoles acquire many of the typical early endosomal markers but not late endosomal/lysosomal ones, suggesting that Mycobacterium arrest maturation before the formation of late phagosomes (12). The active retention of a phagosomal coat protein termed TACO (tryptophan and aspartate containing coat protein) may play an important role in the prevention of phagosome maturation and its consequent fusion with lysosomes (26). As a result, bacteria ensure their survival, but remain in a dormant state producing a latent infection which can last for years.

\subsection{Segregation from the endocytic route}

Chlamydia, Legionella and Brucella phagosomes segregate from the endocytic route to create a suitable niche for intracellular replication. Chlamydia spp. represents one of the most extreme cases. The chlamydiae are obligate intracellular pathogens that grow in cytoplasmic vesicles in a wide variety of host cells, including blood circulating monocytes and tissue macrophages. Chlamydia segregates from the endocytic pathway at a very early stage of development and inhabits membranebound vacuoles termed inclusions. No marker of the endocytic route is accumulated on or within the inclusion (27). These 


\section{DDS against intracellular infections}

inclusions localize close to the Golgi network and fuse to exocytic vesicles containing sphingomyelin, which seems to be essential for the intracellular replication of Chlamydia (28). Furthermore, monocytes have been shown to support C. pneumoniae growth for several days, thus, they can act as carrier systems for the bacteria and spread the infection from the respiratory tract to other organs.

Legionella pneumophila is a facultative intracellular pathogen that colonizes mainly alveolar macrophages. Vacuoles harboring L. pneumophila have also been shown not to acquire endosomal and lysosomal markers (29) but to recruit endoplasmic reticulum (ER)-derived vesicles, indicating that the bacteria reside in a compartment surrounded by ER until the monocytes lyse releasing the bacteria (30). Apparently, a type IV secretion system, the Dot-Icm secretion system, is used by the bacteria to modify the normal organelle trafficking, therefore preventing fusion with lysosomes and permitting L. pneumophila to establish itself within that unique ER-like compartment.

Brucella spp. are facultative intracellular pathogens that infect and replicate inside the cells and organs or the mononuclear phagocytic system (MPS), mainly in the liver and the spleen. When they are captured by phagocytic cells, these organisms present an excellent model for escaping to a safe replicative niche subverting the regular phagosome maturation process. After ingestion the pathogens localizes in early phagosomes. Most of them are in vacuoles retaining late endosomal/lysosomal markers (LAMP-1 positive) and will eventually be killed. However, a few of these vacuoles avoid further fusions with lysosomes. Brucella lipopolysaccharide on the bacterial surface and cyclic beta-1,2-glucan have been proposed to play a role in the control of the phagosomal maturation (31). Cyclic beta-1,2-glucans, structurally related to cyclodextrins, can selectively extract and incorporate cholesterol, glycosylphophatidylinositol and ganglioside GM-1, which are essential for bacterial survival and replication, from lipid rafts to phagosomes. Whatever the case, those vacuoles that successfully evade lysosomal fusion are characterized by the progressive exclusion of LAMP-1 and are capable of interacting with ER exit sites. Subsequently, they fuse to ER to generate ER-derived replicative Brucella-containing vacuoles. In this case, the acidification of the Brucella-containing vacuole is a requisite to trafficking to the ER and subsequent intracellular replication of the bacteria (32).

\subsection{Life within acidic phagosomes}

Staphylococcus aureus is a ubiquitous bacterium that usually produces local infections which spreads to other tissues and organs. Although it has been considered to be an extracellular pathogen, it easily invades non-professional and professional phagocytes. S. aureus persists in the phagolysosomal compartment of phagocytic cells and under these conditions can survive several days before inducing their lysis (22). This particular localization of the bacterium seems to be the reason for the recurrent character of staphylococcal infections, as well as for the failures of clinical treatments using antibiotics that are active in vitro.

Finally, as a new example of intracellular adaptation, the obligate intracellular Coxiella burnetii inhabits an acidified lysosomal-like compartment (18). It has been shown that after its entrance into human macrophages Coxiella adquires late endosomal-early lysosomal markers and resides in acidic vacuoles with $\mathrm{pH}$, remaining metabolically active.

\section{ANTIBIOTIC TREATMENT OF INTRACELLULAR BACTERIAL INFECTIONS}

It seems clear that obtaining a high concentration of a given drug within the cells is not enough to achieve the eradication of intracellular bacteria. Intracellular pathogens, when inside cells, are not only protected from the extracellular environment (antibodies, complement, and even some antibiotics), but also these new physicochemical intracellular conditions induce strategic (structural and metabolic) changes, making them resistant to antibiotics that are active in vitro. Likewise, it should be stressed that the intracellular activity of antibiotics may also change. For instance, the bactericidal activity may be lost at the acidic $\mathrm{pH}$ of phagolysosomes or these drugs may not be able to access the host cell organelles, where the microorganism resides and replicates (see above). Therefore, the final encounter between the pathogen and the antibiotic may not be as successful as predicted by in vitro studies (1).

In order for a treatment to be successful the antibiotic used must fulfill a series of criteria, including the ability to enter and be retained by the cell, the capacity to reach the intracellular target, and the display of activity in that peculiar environment where the bacteria reside.

It has been recognized that a number of antibiotics do not efficiently penetrate cells, thereby achieving low intracellular concentrations. This is the case of beta-lactams and aminoglycosides. Beta-lactam antibiotics exert their antibacterial action by inhibiting the synthesis of peptidoglycan. Most beta-lactams are weak acid compounds that, although capable of crossing biologic membranes, do not accumulate within the cells, probably due both to the more acidic nature of the cell cytosol compared to the extracellular milieu and active antibiotic efflux pumps (33). However, it has been shown that the low accumulation of betalactams in cells can be compensated by their good activity at acidic $\mathrm{pH}$, conferring these drugs therapeutic potential in spite of their apparently unfavorable cellular pharmacokinetics (34).

Aminoglycosides are antibiotics with broad antimicrobial activity, post-antibiotic effect and synergy with beta-lactam antibiotics. However, aminoglycosides show a limited intracellular activity compared to their strong bactericidal activity in an extracellular medium. These drugs diffuse very slowly and poorly through cell membranes due to their high hydrophilicity, but 


\section{DDS against intracellular infections}

they may be incorporated into macrophages using a fluid-phase pinocytosis process when applied at high concentrations and long incubation times. (35). In contrast to the beta-lactams, aminoglycosides are weakly basic, so after reaching the interior of the cell they are confined within the lysosomes, where the acidic $\mathrm{pH}$ may suppress their activity. This lack of activity has been related to the protonation of the molecule at acidic $\mathrm{pH}$. Since aminoglycosides enter the bacteria by active transport, factors affecting this transport would reduce their antibacterial activity (36). Another important issue that limits the use of aminoglycosides is their well-known toxicity. The main adverse effect of aminoglycosides is nephrotoxicity, as a result of renal cortical accumulation, followed by ototoxicity and, to a lesser extent, neuromuscular blockade (37). The narrow therapeutic range between effective and toxic aminoglycoside levels prevents the use of high concentrations of these drugs.

In contrast to the lack of accumulation within the cells reported for the antibiotics mentioned above, macrolides and quinolones present a high cellular accumulation but, nevertheless, there is not always a direct correlation between their accumulation and intracellular activity. Macrolides are weak organic bases that show a marked cell penetration and tissue accumulation, for which they occupy a prominent place in the treatment of infections caused by intracellular bacteria. Inside the cells macrolides distribute mainly to the cytosol and lysosomes where they are trapped by protonation. However, the final outcome depends on the macrolide used. Some studies have shown that azithromycin, for instance, may be essentially bacteriostatic against Listeria monocytogenes (35) and S. aureus (38), probably because their acidic $\mathrm{pH}$ environment decreases macrolides activity (39).

Quinolones, another class of antibiotics that achieve high intracellular concentrations in both infected and non-infected cells, can diffuse to various subcellular compartments without associating with any specific cellular structure (40). The majority of quinolones are effective against intracellular bacteria as Legionella (41) and Listeria monocytogenes (35) but some of them show reduced activity at lower $\mathrm{pH}$, which would explain the discrepancy between their extracellular and intracellular activities when treating pathogens that are localized within phagolysosomes (42).

Furthermore, occasionally, treatment is made difficult due to the specific characteristics of the bacteria, e.g. the presence of metabolically silent or dormant bacteria within the host, which may not be susceptible to some antimicrobial agents. Bacteria in such a phase are much more tolerant to antibiotics than logarithmic phase organisms because they undergo a series of physiological or phenotypic changes that can block antibiotic targets (43). For example, an antibiotic that inhibits protein synthesis will have no effect when the intracellular bacteria are in a dormant stage. Beta-lactams exert their bactericidal activity on multiplying microorganisms (synthesis of peptidoglycan, but not the structure itself), so no effect will be expected if the bacteria do not replicate.

Finally, it has been established that antimicrobial drugs can influence the interaction between microorganisms and phagocytes (44). Antibiotics in general have the ability to alter various functions of the antimicrobial activity of the phagocytic cells of the host. For example, gentamicin enhances the intracellular destruction of microorganisms by the macrophage (45), while the other aminoglycosides inhibit this ability (46). On the contrary, chemotaxis, oxidative burst or cytokine production are not affected by aminoglycosides. Finally, there are studies that indicate that this type of antibiotics does not influence the endocytic machinery, whereas other works suggest inhibitory effects (47).

\section{ANTIBIOTIC ENCAPSULATION IN DRUG DELIVERY SYSTEMS}

In spite of the emergence of new antibiotics, the treatment of intracellular infections remains unsolved. The main challenge for intracellular chemotherapy is to design and develop a carrier system for antibiotics that could be efficiently endocytosed by phagocytic cells and, once inside the cells, capable of releasing the drug. In this sense, polymeric and lipidic DDS are well suited as vehicles for the delivery of antimicrobial agents because they usually provide a sustained drug release effect, minimize the toxicity associated with the encapsulated drugs and increase the overall drug efficacy. Moreover, DDS protect the incorporated drug from premature immunological and enzymatic attacks and, in some cases, they act synergistically with cellular bactericidal mechanisms (48).

\subsection{Liposomes}

Since their discovery in the early 1960s, liposomes have been one of the most extensively studied drug carriers. They are spherical vesicles of one or several concentric lipid bilayers, usually composed of phospholipids and cholesterol, enclosing aqueous compartments (49). Moreover, due to their similarity to biological membranes they present low toxicity and immunogenicity. Liposomes are of interest mainly because of their structural versatility and the possibility of entrapping drugs of different size and solubility properties. Depending on the method used for their preparation, liposomes of different sizes and number of lamellae can be obtained. Based on these parameters liposomes can be classified into small unilamellar vesicles (SUV), large unilamellar vesicles (LUV) and small plurilamellar vesicles (SPLV). Moreover, liposomes are able to entrap both hydrophilic and hydrophobic compounds. Water-soluble compounds are located in the aqueous spaces while lipid-soluble ones are bound or incorporated into the lipid membrane.

When administered intravenously liposomes are rapidly removed by the phagocytic cells of the MPS (50), especially by macrophages in the liver (Kupffer cells) and spleen. This is one of the reasons which have lead to the study of the applications 


\section{DDS against intracellular infections}

of liposomes in the treatment of intracellular infections (51). However, the general use of liposomes as DDS has been hindered by several issues, the most important being the stability of liposomes after their administration. After intravenous administration liposomes are opsonized and exposed to the destabilizing interaction of certain blood constituents, especially lipoproteins. It has been suggested that upon contact between lipoproteins and liposomes, lipoproteins can remove phospholipids from the lipidic structure of the liposomes, living pores through which the content of the liposome is released (52). The grade of destruction of liposomes depends mainly on the type and composition of these vesicles. For instance, SUV composed exclusively of phosphatidylcholine are rapidly disrupted in the serum and lipids from the liposome are transferred to the lipoproteins. Conversely, the incorporation of cholesterol to the formulation can partially inhibit this disintegration (53). Therefore, it seems necessary to be careful in the choice of the liposome components for the successful design and development of liposome-based therapies.

\subsubsection{Uptake of liposomes by mononuclear phagocytic system cells}

Liposomes are mainly taken up by cells by means of endocytosis. However, fusion processes between liposomes and the cell membrane might also be involved in liposome uptake. The internalization of liposomes into phagocytic cells takes place following different steps: stable adsorption to the cell membrane, liposome uptake by an energy-dependent mechanism, the fusion with the lysosomes and degradation of the liposomes by the action of lysosomal enzymes releasing their content (Figure 2) (50). Among these, the first step seems to be the limiting one for liposome internalization, since a stable adsorption of the liposome to the cell surface will further facilitate its uptake. However, the extent of liposome adsorption and subsequent internalization depends on their physicochemical properties.

Allen et al. studied the effect of the physicochemical properties of liposomes on the uptake by murine macrophages and they concluded that small and negatively charged liposomes were the ones most efficiently internalized (54). It seems that the negative charge can enhance the binding of liposomes to macrophages and promote their phagocytosis. Consistent with this, some authors have observed that negatively charged liposomes are internalized slightly more efficiently than neutral liposomes by murine macrophages, and to a greater extent than positively charged liposomes (55). However, it was also found that internalization was higher for antibody-coated liposomes than for non-coated liposomes, probably because in this case the uptake occurs by specific Fc receptor-mediated endocytosis. In addition, this type of coating apparently protects liposomes from cell surface-induced leakage by preventing close contact between the liposome and the cell surface.

Cholesterol may also play an important role in liposome internalization. It has been suggested that the incorporation of cholesterol into liposomes may change the distribution of phospholipids in the membrane rendering phagocytosis more difficult (56). So, although cholesterol concentrations above 50\% give optimal stability to liposomes in the presence of serum lipoproteins, a lesser amount of cholesterol would be preferable to provide sufficient liposome stability without compromising capture by phagocytic cells (57).

Nevertheless, as stated above, liposomes are finally found at the lysosomal compartment,hence this strategy seems to be useful to fight bacteria that reside in lysosomes. Conversely, when the bacteria localize within other subcellular compartments the final encounter between the pathogen and the drug may be impaired. Different formulation strategies have been developed to solve this problem, including the preparation of $\mathrm{pH}$-sensitive liposomes, which have the ability to escape from the endosomal compartment and release the encapsulated active compound in the cell cytoplasm.

\subsubsection{Intracellular destination of $\mathrm{pH}$ sensitive liposomes}

$\mathrm{pH}$ sensitive liposomes are stable at neutral $\mathrm{pH}$ but are destabilized at mildly acidic $\mathrm{pH}$ delivering the encapsulated material into the cell cytoplasm. There are two main categories of these liposomes: intrinsically pH-sensitive liposomes and those which utilize an external non-lipid trigger. In general, it is assumed that $\mathrm{pH}$-sensitive liposomes are taken up more efficiently than $\mathrm{pH}$-insensitive liposomes (58). After binding to the cell surface, liposomes enter cells via endocytosis and then are delivered into endosomes, where they encounter a more acidic environment than the extracellular milieu. This type of liposomes presents membranes that have been designed to destabilize and/or fuse with the endosomal membrane when the $\mathrm{pH}$ value drops, resulting in the release of the active compound to the cytoplasm prior to arrival in the lysosomes (Figure 2). Dioleoyl phosphatidylethanolamine (DOPE) and weakly acidic amphiphilies have been widely used to confer intrinsic $\mathrm{pH}$-sensitivity to liposomes $(58,59)$. At physiological $\mathrm{pH}$, amphiphilies possess negative charges that stabilize the lipidic bilayer structure. However, as the $\mathrm{pH}$ decreases (as it happens in endosomes), the head group of amphiphilies become protonated and phosphatidylethanolamine molecules revert from a bilayer phase to a non-bilayer phase, resulting in liposomal destabilization. Another strategy for endosomal escape of the pH-sensitive liposomes is the addition of proteins or peptides that exhibit membrane lytic or fusion properties at acidic $\mathrm{pH}$. For example, the listeriolysin $\mathrm{O}$ from Listeria monocytogenes has been studied for this purpose (60).

As with conventional liposomes, the major limitation of $\mathrm{pH}$-sensitive liposomes in vivo is their aggregation and instability in plasma, but this problem can be solved by adding polyethylene glycol to the formulation (59). This coating prevents interaction with blood constituents, leading to an increased stability in plasma and also to a lower recognition by circulating monocytes. 


\section{DDS against intracellular infections}

\subsubsection{In vitro efficacy of liposome-assisted antibiotic treatment}

Several antibiotics have been encapsulated into liposomes to improve their interaction with phagocytic cells and obtain an intracellular accumulation (61). Most studies on liposome-encapsulated antibiotics have been performed in Mycobacteriuminfected cells. In this sense, liposome-entrapped quinolones and macrolides have shown to enhance the killing of intracellular $M$. avium complex (MAC). Encapsulating ofloxacin and clarithromycin into liposomes significantly increased the internalization and intracellular efficacy of the drugs against MAC in human macrophages (62). Thus, at day 4 following treatment liposomeencapsulated antibiotics were able to reduce the number of colony forming units (CFU) by more than 0.93 log units and 0.4 logs, respectively, in comparison to free drugs. Similarly, liposome-encapsulation of azithromycin, rifabutin and clarithromycin significantly increased their delivery into macrophages and their antimycobacterial activity, compared to the same concentration of the free drugs (63). In these two studies, the addition of ethambutol to either the free or liposome-encapsulated drugs led to an enhancement of their activity.

Azithromycin- and ciprofloxacin-containing liposomes presented 40-fold greater activity in murine macrophages harboring Mycobacterium avium (64). Among the different ciprofloxacin formulations tested, a maximal cellular accumulation and antimycobacterial activity of ciprofloxacin was reached as the negative charge of the liposomes increased. The quinolone sparfloxacin has also been entrapped into liposomes in order to be used against MAC-infected J774 macrophages (65). However, the reduction of the intracellular bacteria was only slightly higher for the liposome-encapsulated antibiotic in comparison to its free form. In this case, the lack of a marked increase in the effectiveness of the liposomal form of sparfloxacin may be ascribed to its intrinsic lipophilic nature, which enables it to accumulate efficiently in macrophages.

Aminoglycoside antibiotics have also been tested for their ability to reduce intracellular MAC (66). Liposomal streptomycin was more effective in the intracellular killing of bacteria than the free drug, achieving a reduction of $89.1 \%$ of the CFU with the higher dose used $(50 \mathrm{mg} / \mathrm{l})$. It was found that the unloaded liposomes reduced the infection by 0.2 log units at this lipid dose, suggesting that they could improve the antibacterial activity of the antibiotic. The same study also reports that $5 \mathrm{mg} / \mathrm{l}$ of ciprofloxacin-loaded liposomes produced a 50-fold reduction of the initial level of infection compared to the free drug.

Resorcinomycin A is another type of antibiotic that has shown more effective intracellular activity against MAC when its liposomal form is used (67). Three days after treatment with a $50 \mathrm{mg} / \mathrm{L}$ dose of free and liposome-encapsulated antibiotic resulted in $62 \%$ and $93 \%$ of bacterial growth inhibition, respectively. Moreover, liposomal resorcinomycin A maintained its antimycobacterial activity for 7 days after treatment, while the activity of the free drug lasted for less than 3 days.

Liposomes containing aminoglycosides have also shown to enhance the killing of other intracellular bacteria. Vitas et al. found that at a $20 \mathrm{mg} / \mathrm{L}$ concentration positively-charged SPLV containing $30 \%$ cholesterol and gentamicin completely eradicated the Brucella abortus infection in murine macrophages (57). In contrast, the same vesicles with 50\% cholesterol had a markedly lower intracellular activity, reducing the number of CFU by $0.84 \log$ units compared to the untreated control group. Gentamicin has also been entrapped into $\mathrm{pH}$-sensitive liposomes in order to be used against Salmonella typhimurium and Listeria monocytogenes (68). At an equivalent gentamicin dose, the liposomal carriers achieved and intracellular gentamicin concentration 21.5 times higher than the free drug, being those liposomes that included DOPE and N-succinyl-DOPE in the formulation the most effective. This antibacterial activity was especially greater against L. monocytogenes and a hemolysinexpressing S. typhimurium, which resides in the cell cytoplasm, than against wild-type $S$. typhimurium, suggesting that the gentamicin released from $\mathrm{pH}$-sensitive liposomes concentrates mainly in the cell cytoplasm. In another study, liposomes containing dihydrostreptomycin showed 40 -fold higher activity against intracellular $S$. aureus in murine macrophages in comparison to the effect exerted by the drug solution and unloaded liposomes (69).

The entrapment of the beta-lactam antibiotic, flucloxacillin, and rifampicin in liposomes also improved the intracellular activity of these antibiotics against $S$. aureus compared to the free drugs (70). The study was performed in phagocytes which had a decreased or lack of ability to produce reactive oxygen intermediates (ROI). The number of surviving bacteria after the treatment with liposome-encapsulated flucloxacillin and rifampicin was 5 and 2.5 times lower than when treated with the free drugs.

Finally, beta-lactam antibiotics have also been liposome-encapsulated in order to treat intracellular infections produced by Listeria monocytogenes. A $50 \mathrm{mg} / \mathrm{L}$ concentration of ampicillin entrapped in liposomes killed $90 \%$ of the intracellular bacteria after $6 \mathrm{~h}$ incubation (71). On the other hand, penicillin G-containing liposomes reduced the infection by more than 3 log units when $100 \mathrm{U} / \mathrm{mL}$ of entrapped penicillin $\mathrm{G}$ were added to the infected cell culture (72).

\subsection{Polymeric micro- and nanoparticles}

Microparticles and nanoparticles were developed as alternative systems to liposomes, to solve their stability problems during storage and after administration in biological fluids. This improved stability and the possibility of obtaining a modulable controlled release of the encapsulated drug are the main advantages of polymeric particles over liposomal carriers. Thanks to advances in techniques for microencapsulation and the development of new polymers, such vectors are currently the subject of an extensive research on the encapsulation of many active ingredients such as hormones, anticancer antigens and antibiotics (73). 


\section{DDS against intracellular infections}

In the last few decades several methods were developed to prepare polymeric micro- and nanoparticles (74). Some of the common methods used for polymeric particle formulation are emulsification-solvent removal (or solvent evaporation), phase separation (or coacervation), interfacial polymerization and spray-drying. The selection of a particular technique depends mostly on the physicochemical characteristics of the drug of interest. In general, the polymers used for drug encapsulation purposes can be broadly divided into two groups, depending on their nature: natural and synthetic polymers. Natural polymers (i.e. human or bovine serum albumin, gelatin, collagen, alginate, chitosan, hyoluronan, starch) are polymers derived from natural sources. Their lack of purity and homogeneity and the risk of disease transmission have led to a decrease in their use. On the other hand, synthetic biodegradable and biocompatible polymers (i.e. poly(a-hydroxyacids), polyanhydrides like poly(sebacic acid) and poly(fatty acid dimer-sebacic acid), are extensively used for the encapsulation of many drugs. Among these synthetic polymers, the aliphatic polyesters poly(lactic acid) (PLA), and copolymer poly(lactic co-glycolic acid) (PLGA) are the most widely investigated class of polymers with regard to toxicological and clinical data (75). PLA and PLGA are non-toxic, biocompatible and biodegradable polymers approved by the Food and Drug Administration for human consumption $(76,77)$.

\subsubsection{Endocytosis and the intracellular fate of polymeric particles used as drug delivery systems}

Phagocytic cells are programmed not only to recognize pathogens, but also to localize and readily internalize foreign materials such as, for instance, polymeric particles used as DDS (78). Upon their intravenous administration, the particles interact with blood cell constituents and are generally captured by the macrophages of the MPS, being rapidly cleared from the bloodstream. This phenomenon is related to opsonization, which involves the adsorption of plasma proteins, complement components and factors among others. Therefore, this fact, which would be a disadvantage in the treatment of some infections, provides passive targeting to the interior of monocytes and turns polymeric particles into exceptional candidates for the treatment of intracellular infections that affect the MPS.

As illustrated above in the context of pathogens, the first experimental observation concerning intracellular transport of polymer particles was the fact that there is also a wide variation in the reported rates of phagosome maturation depending on the particles used $(5,79)$. The receptors or mechanisms of entry used during phagocytosis undoubtedly have major consequences on the biochemical nature of the nascent phagosomes (80). Namely, the physico-chemical parameters of the particles, such as size and surface hydrophobicity, determine some of the characteristics of the initial endosomal compartment (81). Further fusions with other endocytic vesicles and recruitment of various proteins from the cytosol influence particle trafficking along the phagolysosome pathway $(3,81)$ (Figure 3). However, the exact mechanism mediating the cellular uptake of particles and the advantages of specific sizes and surface properties of these DDS are just beginning to be understood.

It is generally assumed that hydrophobic particles are more easily phagocytosed than hydrophilic ones by forming hydrophobic interaction with the cell surface (82). However, it has been shown that $1 \mu \mathrm{m}$-sized hydrophobic latex beads remain associated with early endosomes but do not fuse with lysosomes, finally being found in a relatively neutral environment $(\mathrm{pH} 6.0$ to 6.8). It seems that the tight apposition displayed with regard to the phagosome membrane prevents phagosome from its maturation, which is similar to the process observed with Mycobacterium to inhibit phagosome-lysosome fusion (83). On the other hand, hydrophilic particles are degraded at a faster rate upon the phagosome maturation into phagolysosomes. These particles are then found in the more acidic microenvironment typical of matured phagosomes ( $\mathrm{pH} 4.6$ to 5.1) (81).

According to the size, Koval et al. (84) found that particles presenting a size below $1 \mu \mathrm{m}$ are internalized by clathrinmediated endocytosis. Conversely, as the size of the particles increases, the uptake process becomes more clathrin-independent. Thus, for particles with a diameter of $1 \mu \mathrm{m}$ or above, internalization occurs by phagocytosis after the formation of actin-coated invaginations in the macrophage membrane. In this study it was also observed that the mechanism of entry further influenced particle behavior inside the cell. Following their uptake, micrometric-range latex beads were quickly delivered to lysosomes while particles of smaller size showed a delayed delivery to lysosomes. Once there, it is expected that drug delivery particles can be degraded and the encapsulated drug released.

Thus, in general, the more a particle is diverted from endocytic trafficking into phagolysosomes, the more slowly its contents are degraded. This behavior could be exploited to increase the half-life of therapeutic molecules.

\subsubsection{Synergy of polymeric particles with cellular bactericidal mechanisms}

Another point to be considered regarding the treatment of intracellular infections is the synergy of the polymeric particles with the bactericidal mechanisms of the phagocyte. The stimulation of the intracellular ROI may act synergistically with the antibiotic activity to kill intracellular bacteria, thus increasing treatment efficiency. For example, Lecároz et al. studied the effect of different PLGA polymers (PLGA 502H and 752H) on the characteristics of gentamicin-containing microparticles (85). The main difference between these two polymers is the lactide/glycolide ratio, being 50:50 for the copolymer 502H and 75:25 for $752 \mathrm{H}$. In addition, the copolymer $752 \mathrm{H}$ has a higher molecular weight and is more hydrophobic compared to $502 \mathrm{H}$. These authors found that microparticles of uncapped PLGA 502H (free -OH and $\mathrm{COOH}$ groups) with hydrophilic end-groups stimulate the oxidative burst of macrophages in a more efficient way (measure of general cell activation) (86). However, the phagocytosis of microparticles may not correlate with the activation of the cells. Overall, the phagocytosis of microparticles depends mainly on the hydrophilicity of the polymer, whereas it is the presence of uncapped end-groups which influences the cell activation (87). Smaller and less hydrophilic PLA and PLGA 752 particles may be efficiently internalized, but their surface characteristics may 


\section{DDS against intracellular infections}

not be able to activate the oxidative metabolism to an extent comparable to that of polymer surfaces containing specific functional groups, such as $-\mathrm{COOH}$ and $-\mathrm{OH}$ in the end-group of uncapped polymers (88).

These results were corroborated in experiments performed with macrophages infected with Brucella. It was found that the effect of gentamicin-loaded PLGA 502H microparticles significantly decreased the intracellular bacterial levels of infected monocytes in comparison to PLGA 502 microparticles, although PLGA 502H released only 14\% of the encapsulated gentamicin within the initial $24 \mathrm{~h}$ in vitro, as compared to a 50\% burst release from the PLGA 502 microparticles (89). It can thus be hypothesized that a higher number of PLGA $502 \mathrm{H}$ particles were phagocytosed, increasing bactericidal oxidative burst that will act synergistically with gentamicin activity (48).

Recently, this potential capacity of polymeric DDS to induce the activation status of the macrophages has also been confirmed by Sharma et al. in the case of PLA microparticles (90). In this study, the authors observed that microparticles containing two anti-tuberculosis agents, rifampicin and isoniazid, efficiently targeted lung macrophages and enhanced the respiratory burst and nitric oxide production by macrophages. The results showed significantly higher amounts of reactive nitrogen intermediates in $M$. tuberculosis-infected cells treated with inhalable microparticles in comparison to non-treated ones. Moreover, drug-containing microparticles induced the release of $\mathrm{O}_{2}^{-}$and both TNF-alpha and IL-12 cytokines in contrast to unloaded microparticles.

Taken together, these studies reveal that the ingestion of microparticles by macrophages may result in their activation and, subsequently, may enhance the host defense functions of the immune system.

\subsubsection{In vitro efficacy of polymeric particles for antibiotic treatment}

In vitro studies using infected macrophages have demonstrated that antibiotic-containing polymeric particles efficiently target phagocytic cells achieving therapeutic drug concentrations intracellularly and consequently improving the killing of intracellular microorganisms. PLGA microparticles containing gentamicin have been shown to enhance the intracellular killing of Brucella abortus (89) and Brucella melitensis (91) in infected macrophages. Prior et al. prepared gentamicin containing microparticles by the spray-drying method using PLGA 502 and PLGA 502H polymers (89). Treatment of the infected cells with gentamicin-loaded PLGA 502 and PLGA 502H microparticles reduced the infection by 1 and $2 \log$ units, respectively, compared with untreated controls, whereas unloaded microparticles were not able to enhance the killing of bacteria. However, only the treatment with gentamicin PLGA 502H microparticles was significantly more effective in reducing bacteria than free gentamicin. Furthermore, in order to improve the dispersability of the particle the effect of the addition of $2 \%$ (w/v) of poloxamer 188 to the medium was studied. Interestingly, it was found that an increased antibacterial activity of microencapsulated gentamicin is achieved by dispersing the microparticles in this surfactant (3.5 log units for PLGA 502H microparticles). Although the mechanism responsible for this effect is still unclear, several factors may have played a role in this outcome. Surfactant adsorption onto the microparticles may have altered the surface polarity and subsequently enhanced cell adhesion and phagocytosis, which might have been additionally facilitated by a higher degree of particle dispersion in the presence of the surfactant. One can also speculate that unspecific cell activation by the surfactant and synergistic enhancement of the antibiotic activity could also have exerted some antibacterial effect.

PLGA $502 \mathrm{H}$ microparticles prepared by the solvent evaporation method were further used against $B$. melitensis infected human macrophages (91). The results demonstrated that these PLGA microparticles are efficiently captured by the macrophages and that the gentamicin released from these particles is active and can exert its bactericidal effect inside the macrophagic cells. A $30 \mu \mathrm{g}$ dose of encapsulated gentamicin reduced the intracellular Brucella counts by 2.2 log units. By using transmission electron microscopy and immunocytochemistry (gold-labeled antibodies against gentamicin), it was possible to observe the released of the antibiotic from the particles, not only in the cytoplasm and nucleus but also inside Brucella's compartment (Figure 4). PLGA 502H nanoparticles were also prepared by this method, but even though they showed a higher capacity for monocyte activation than microparticles, they were discarded for in vitro efficacy studies due to the low antibiotic entrapment obtained (92).

Another aminoglycoside, streptomycin, and the tetracycline doxycycline have also been encapsulated in polymeric nanoparticles and used against intracellular B. melitensis (93). The antibiotics were simultaneously incorporated in a PEO- $b$ PtBA (poly(ethylene oxide)-b-poly(tert-butyl acrylate)) diblock copolymer and efficacy studies were performed in infected murine macrophages. Both the antibiotic combination containing nanoparticulated carriers and the free drugs completely eradicated the infection. Doxycycline has the ability to penetrate and concentrate into cells but, due to its mainly bacteriostatic activity for the treatment of brucellosis, it is used in combination with an aminoglycoside presenting bactericidal activity. In this case, the capability of the polymeric nanoparticles to enter cells and target intracellular B. melitensis was confirmed. Moreover, as discussed below, although there were no differences between the efficiency of free and encapsulated drugs in vitro, studies in vivo confirmed the advantage of using encapsulated gentamicin over free gentamicin (93).

Encapsulation in polyisohexylcyanoacrylate (PIHCA) nanoparticles is a different well-studied strategy for the delivery of another antibiotic, ampicillin, to mouse macrophages (94). It has been observed by confocal micrographs and transmission electron microscopy that ampicillin-loaded PIHCA nanoparticles efficiently target Salmonella-infected murine macrophages. 


\section{DDS against intracellular infections}

Although at early stages, endocytic vesicles contained mainly intact nanoparticles, at later stages nanoparticles were localized in close contact with bacteria inside phagosomes and phagolysosomes. Moreover, ultrastructural autoradiography showed that, apart from increasing the penetration of the antibiotic into macrophages, ampicillin nanoparticles had increased bactericidal effect against Salmonella typhimurium, even at incubation times as short as 2-4 h (95).

Ampicillin bound to PIHCA nanoparticles has also been studied in the same in vitro model of L. monocytogenes infection (96). In addition to the results obtained for Salmonella these nanoparticles were also effective regarding the treatment of L. monocytogenes-infected cells. After $30 \mathrm{~h}$ of incubation, nanoparticles reduced the number of viable bacteria by $1.75 \mathrm{log}$ units over the free drug. However, in this case a lag period of 6-9 $\mathrm{h}$ was required for the antimicrobial effect to begin, probably due to the different intracellular localization of these bacteria.

\section{TREATMENT OF EXPERIMENTAL INFECTIONS MEDIATED BY DRUG DELIVERY SYSTEM}

\subsection{Tuberculosis}

Tuberculosis is one of the leading causes of death worldwide with more than 3 million deaths annually. It is estimated that one-third of the world's population is infected with Mycobacterium tuberculosis, the causative agent of tuberculosis, Asia and Africa being the continents with the highest annual incidence. Moreover, with the increased incidence of acquired immunodeficiency syndrome, tuberculosis has become a significant opportunistic disease.

Current treatment of tuberculosis requires daily administration of multiple antitubercular drugs for at least 6 months. This prolonged and uncomfortable therapeutic schedule is the main reason for patient non-compliance and therefore for therapeutic failures and the emergence of multidrug resistant-tuberculosis.

In this context, the development of a drug carrier that, with a single dose, could maintain therapeutically active levels of the drug for prolonged periods of time without causing any toxicity would be the ideal solution to tuberculosis treatment. Some of the most important achievements of the encapsulation of antitubercular drugs are the reduction of drug-associated toxicity, as well as the reduced dose and dosing frequency needed to achieve a therapeutic effect. The first objective of this DDS-mediated therapy is to achieve sustained therapeutic drug levels at the infection sites. In this sense, important improvements have been obtained. When administered as conventional formulations, rifampicin and isoniazid achieve low serum concentrations and are maintained in circulation for less than $24 \mathrm{~h}$. Conversely, intravenous administration of $12 \mathrm{mg} / \mathrm{kg}$ and $10 \mathrm{mg} / \mathrm{kg} \mathrm{liposome-}$ entrapped isoniazid and rifampicin resulted in sustained therapeutic drug levels for up to 5 and 7 days in serum and organs respectively (97). This modification of the pharmacokinetics of these drugs may permit a weekly treatment regimen instead of the current daily one. Moreover, the chemotherapeutic efficacy of liposome-encapsulated isoniazid and rifampicin administered once a week has been demonstrated in a murine tuberculosis model. It was found that only one third of the dosage of isoniazid and rifampicin was necessary to achieve therapeutic effects when administered as a liposomal formulation.

At equivalent doses, antibiotic-loaded liposomes were significantly more effective in reducing the number of bacteria in lungs, liver and spleen than the combination of free oral drugs. Besides, no sign of liposome-induced hepatotoxicity was observed during the treatment. Thus, this liposome-mediated treatment of tuberculosis may be a promising approach to obtain a therapy with good patient compliance, low cost and reduced dosage frequency and toxic effects.

The encapsulation of antitubercular drugs in polymeric particles is another strategy to improve the current therapeutic regimen of tuberculosis. In the last few years several antitubercular drugs-containing PLGA and PLA microparticles and mainly nanoparticles have been exhaustively studied. One of the most novel proposals for the chemotherapy of tuberculosis is the use of inhalable or respirable particulate carriers that deliver antimycobacterial drugs directly in the lungs, thus achieving high drug concentration at the main infection site while reducing systemic toxicity. The pharmacokinetics and antimycobacterial effect of polymeric particles carrying antitubercular drugs have been studied in guinea pigs. Polymeric carriers have shown better pharmacokinetics profiles than liposomes. A single nebulization of rifampicin, isoniazid and pirazinamide coencapsulated in PLGA nanoparticles administered to guinea pigs maintained therapeutic drug concentrations in the plasma and lungs for 6 to 8 days, and for up to 11 days, respectively (98). Moreover, great antimycobacterial activity was shown in the lungs of the animals treated with nanoparticles. The nebulization of the nanoparticulated formulation every 10 days resulted in the sterilization of the lungs after five doses. In contrast, 46 daily doses of orally administered drugs were required to achieve an equivalent therapeutic outcome. In this case, the superior efficacy of nanoparticulate systems over the microparticulated ones seems to be clear, as no inhalable microparticle formulation has been able to completely eradicate the lung infection $(99,100)$.

Another interesting feature is that the stability of polymeric particles allows the possibility of an oral administration. U1-Ain and co-workers demonstrated that PLGA microparticles provide a sustained release of isoniazid, rifampin and pyrazinamide for up to 9 days in various organs and for at least 3 days in plasma (101). In this case, weekly oral administration of nanoparticles showed similar results to those obtained by the daily-administration of free drugs. Moreover, the chemotherapeutic efficacy of PLGA-encapsulated drugs given orally was equivalent to that of subcutaneously injected drugs, but with the advantage of being a more comfortable administration route. Once again the same drugs rendered better results when encapsulated into nanoparticles versus microparticles. Following a single oral administration of these nanoparticles in mice, drug 


\section{DDS against intracellular infections}

levels could be detected in plasma for 6-9 days and in organs for 9-11 days. Further, a total of 5 doses of drug-loaded nanoparticles administered every 10 days to Mycobacterium tuberculosis-infected mice completely eradicated the bacteria on the infected tissues (102). Thus, these studies clearly document the efficacy of polymeric nanoparticle-based antituberculosis chemotherapy in experimental tuberculosis as an alternative to long-term treatment regimens.

\subsection{Brucellosis}

Human brucellosis is still a major health problem worldwide with more than 500,000 cases per year. While the endemic disease is limited to some areas of the Mediterranean basin and developing countries in Asia, Africa and Latin America, the current expansion of international travel increases the likelihood that the disease may be observed in any non-endemic area.. Brucellosis is a systemic infection that can involve any organ or tissue in the body. The onset may be acute with fever, sweats, fatigue, malaise and arthralgias, or even more insidiously, may develop over a period of weeks to months. Most relapses appear during the first six months after therapy with milder clinical findings than those usually observed at the initial episode of the disease, although some patients develop particularly insidious clinical relapses.

The aim of any treatment for human brucellosis is to fight against the symptoms of the disease, reduce complications and, eventually, prevent relapses. However, since the complete eradication of the microorganisms is very difficult to achieve, synergistic or additive antibiotic combinations are required for prolonged periods of time to obtain satisfactory results.

Although in the last two decades many trials have provided good evidence criteria for its antibiotic treatment, the most appropriate antimicrobial therapy for human brucellosis continues to be a controversial issue. Overall, the combination of oral doxycycline and intramuscular aminoglycosides is considered the most effective treatment, with an associated relapse rate of $5 \%$ (103). Significant drawbacks include the need for parenteral administration and the potential toxicity of aminoglycosides. The effective role of aminoglycosides in this combination should be stressed: whereas aminoglycosides used as monotherapy have shown little efficacy, probably due to their poor intracellular penetration, they have shown a substantial synergistic effect with tetracyclines in vitro and in vivo (104). The oral combination of doxycycline and rifampicin for 45 days is so far the best alternative treatment, because of its lack of side effects and its acceptability to patients. However, this treatment it is less effective (failure rate about $15 \%$ ), mainly in the complicated forms of the disease (103).

Thus, alternative methods such as DDS to achieve high intracellular bactericidal activity should be considered. The development of antibiotic-containing liposomes was the first approach to overcome the mentioned drawbacks and to obtain a more effective and compliable treatment for brucellosis. Vitas et al. designed a new method for the preparation of cationic SPLV loaded with gentamicin, which achieved a high removal of intracellular bacteria (57). Subsequent in vivo studies in mice infected with $B$. abortus concluded that these liposomes produce a fairly significant protective effect, not observed in antibiotic-free treatments or with negatively charged liposomes, demonstrating the efficacy of those newly developed liposomes in the treatment of brucellosis (105). However, despite the promising results described above, important drawbacks are attributed to these vesicles, such as their instability in the presence of blood lipoproteins and their osmotic fragility, which can destabilize them, leading to the leakage of the entrapped drug. In addition the long-term stability during storage of liposomes is also an unresolved problem.

Therefore, several works with polymeric microparticles and nanoparticles were initiated. Prior et al. prepared PLGA $502 \mathrm{H}$ microparticles by the spray-drying method. Vectors with a good encapsulation and release profile of gentamicin were obtained. In addition, the formulations activated macrophages (48) and significantly reduced the number of intracellular bacteria in vitro (89) Unfortunately the particles lacked therapeutic activity in vivo because, although their size was suitable for intravenous administration, these formulations showed a marked tendency to aggregate, resulting in poor circulation of the microparticles to the spleen, considered the target organ of Brucella.

The particle aggregation was solved by using the emulsion formation/solvent evaporation method when formulating particles. In fact, Lecároz et al. showed that when administered in $502 \mathrm{H}$ micro or nanoparticles or $752 \mathrm{H}$ microparticles, gentamicin was successfully delivered to the liver and the spleen $(85,92)$. Pharmacokinetic parameters illustrated the markedly altered distribution of PLGA-loaded gentamicin compared to the free drug, as higher concentrations of the antibiotic in the spleen and liver were observed when it was administered loaded in microparticles. At the same time, undetectable concentrations were obtained in the serum samples, precluding drug accumulation in the kidneys.

This long persistence of gentamicin beyond the half-life of the particles is probably a result of the nature of this aminoglycoside. These drugs are highly stable and are not metabolized in the liver. As a consequence, their intracellular retention is very high.

Furthermore, when mice were chronically infected with a virulent strain of this bacterium and treated with both microparticle formulations, the level of infection in the spleen was significantly reduced after a single microparticle administration $(1.45 \log$ for $752 \mathrm{H}$ microparticles and $0.45 \log$ for $502 \mathrm{H}$ microparticles, at 3 weeks post-treatment). In contrast, free gentamicin was ineffective. Distribution studies showed that after two weeks, only $752 \mathrm{H}$ microparticles remained intact in the spleen and, in modest quantities, in the liver. However, gentamicin was detected up to four weeks post-administration in both 


\section{DDS against intracellular infections}

spleen and liver after a single dose of administration of the microparticle formulations. These gentamicin concentrations were within the range of the in vitro $\mathrm{MBC}$ for Brucella, and consequently a significant reduction of the level of infection was observed in the spleen of mice chronically infected, 7 days after a single microparticle administration.

Recently, polymeric nanoparticles prepared using PEO-b-PtBA diblock copolymer and carrying streptomycin and doxycycline have been developed for brucellosis treatment as alternative systems to PLGA copolymers (93). B. melitensisinfected mice were treated with $9 \mathrm{mg} / \mathrm{kg}$ streptomycin and $1.8 \mathrm{mg} / \mathrm{kg}$ doxycycline, either free or incorporated in nanoparticles. After 3 days, both the free drugs and nanoparticles containing the drug combination had reduced the infection in the spleen $(0.51$ $\log$ and $0.72 \log$ reduction, respectively) but only the nanoparticles obtained a significant reduction of the infection in the liver (0.79 log reduction). Thus, alternative methods such as DDS to achieve high intracellular bactericidal activity seem promising. The possible use of drug delivery systems containing aminoglycosides may be one of the most relevant therapeutic advances in human brucellosis treatment in the recent years.

\subsection{Salmonellosis}

Salmonellosis is one of the most serious food-borne diseases affecting humans. It may be considered the most important pandemic zoonosis under natural conditions. Salmonella spp. are facultative intracellular bacteria that can cause a wide spectrum of disease ranging from gastroenteritis, enteric fever, bacteremia and focal infections, to a chronic carrier state. Currently, the most important problems concerning treatment of salmonellosis are the emerging resistance to fluoroquinolones, the production of extended-spectrum beta-lactamases and the increase of multidrug resistant Salmonella strains.

Several studies using antibiotic-loaded nanoparticles have been performed in order to assess the suitability and efficacy of these carriers in experimental models of salmonellosis. PIHCA nanoparticles have shown promising results, reducing the mortality in acute infections caused by S. typhimurium in mice (106). A single dose of $0.8 \mathrm{mg}$ encapsulated ampicillin resulted in the survival of all mice 60 days after bacterial inoculation. In contrast, 3 doses of $32 \mathrm{mg}$ free ampicillin were required to obtain the same effect. All untreated mice and those treated with empty nanoparticles died within 10 days post infection. The authors attribute this enhanced effect to the selective targeting of the carriers to the infected tissues as well as to the effective intracellular targeting observed in vitro $(94,95)$. In order to assess whether polyalkycyanoacrylate nanoparticles were also effective against non-dividing bacteria, Page-Clisson et al. studied the effectiveness of these carriers in a model of persistent S. typhimurium infection (107). They found that although at early stages of the infection, when bacteria are actively dividing, there was an antibacterial effect, neither free nor nanoencapsulated ciprofloxacin or ampicillin could significantly reduce infection in the liver or the spleen at later stages. Therefore, the important role of the metabolic state of the bacteria on their susceptibility to antibiotics was again confirmed.

Ampicillin has also been entrapped in liposomes for the treatment of salmonellosis. As seen with nanoparticles, ampicillin-containing liposomes concentrate mainly in the spleen and at a lesser extent in the liver. However, liposomes were shown to be less effective for the treatment of acute S. typhimurium-induced samonellosis than PIHCA nanoparticles (108). A single dose of $0.8 \mathrm{mg}$ liposome-bound ampicillin protected $60 \%$ of the treated mice in comparison with $100 \%$ protection achieved with nanoparticles (106). Moreover, the nanoparticles were more effective targeting ampicillin to the liver than liposomes.

Apart from ampicillin, aminoglycoside-loaded liposomes have also been employed in the treatment of experimental salmonellosis. A $20 \mathrm{mg}$ dose of liposome-entrapped streptomycin was shown to prolong survival of mice to at least one month. When 2 doses of $20 \mathrm{mg}$ or a single dose of $80 \mathrm{mg}$ were administered, the mice survived for more than 55 days (109). Moreover, the liposome entrapment of streptomycin markedly decreases its toxicity. Gentamicin is also more effective against $S$. typhimurium and S. dublin when it is administered bound to liposomes. $10 \mathrm{mg} / \mathrm{kg}$ liposomal gentamicin markedly enhanced the survival of S. typhimurium-infected BALB/c mice regardless of whether liposomes were administered a week prior or 1 or 2 days after infection (110). In the case of mice bearing a systemic $S$. dublin infection, the administration of 2, 10 and $20 \mathrm{mg}$ gentamicin loaded into liposomes resulted in 80,90 and 100\% mice survival, respectively (111). pH-sensitive liposomes have also been successfully used for the treatment of S. typhimurium-infected mice (112). Gentamicin concentration in the liver and spleen were increased by 153- and 437-fold, respectively, when this drug was administered encapsulated into pH-sensitive liposomes. Moreover, the concentration of the antibiotic in the kidney was reduced 2.8 times. This favorable pharmacokinetic behavior was associated with an improved in vivo efficacy of the carriers. The administration of 0.2 to $5 \mathrm{mg} / \mathrm{kg}$ liposomes resulted in a 3 log reduction of the bacteria in the spleen, while the same doses were ineffective when administered as free drugs.

Therefore, alternative methods such as DDS which achieve high protective and bactericidal activity should be taken into account in the future as suitable treatments for Salmonella-induced infections.

\subsection{Listeriosis}

Listeriosis is a severe foodborne disease caused by the facultative intracellular pathogen, Listeria monocytogenes. This bacterium is an important cause of life-threatening infections, including sepsis and meningoencephalitis, especially in neonates, pregnant women, the elderly and immunocompromised patients. Additionally, although isolates of L. monocytogenes are susceptible to a wide range of antibiotics (35), the clinical management of listeriosis remains a difficult task due to the ability of 


\section{DDS against intracellular infections}

this microorganism to multiply intracellularly and spread cell-to-cell without leaving the protective environment of the host's cells. The current therapy of choice for listeriosis treatment is a combination of ampicillin and an aminoglycoside, usually gentamicin (113).

Different carriers of ampicillin have been developed in order to increase the antibiotic availability at the intracellular sites where the bacteria reside. Distribution studies in uninfected mice showed that when ampicillin was administered intravenously entrapped into liposomes, it quickly concentrated in the liver and mainly in the spleen (108). Moreover, the efficacy of liposomes and free antibiotic were assessed in Listeria-infected mice. Seven days after the treatment, ampicillinloaded liposomes had reduced the infection by $3.2 \mathrm{logs}$ in the liver and $2.8 \mathrm{logs}$ in the spleen, while free ampicillin was ineffective. In another study, Bakker-Woudenberg et al. also observed that higher concentrations of ampicillin were obtained in the liver when the antibiotic was administered bound to liposomes (71). Moreover, they found that the lipid composition of the carriers had a marked influence on the in vivo behaviour of the liposomes. For example, although less fluid liposomes maintained higher ampicillin concentration for a longer period of time in the liver, they were less effective in the killing of bacteria than more fluid liposomes.

Regarding nanoparticles, ampicillin-containing polymeric nanoparticles were also significantly more effective than free ampicillin in a chronic experimental listeriosis in athymic nude mice (114). Seven days after the treatment administration, ampicillin-bound nanoparticles completely eradicated the infection in the liver, while free ampicillin did not ensure liver sterilization during the 19 days that the experiment lasted, even with doses as high as $48 \mathrm{mg}$ (this total dosage was divided into three doses of $16 \mathrm{mg} / \mathrm{each}$ ). Regarding the spleen, the differences between these treatments were not so notorious, but the ampicillin-loaded nanoparticles significantly reduced the infection compared to the untreated group (2.1 log reduction), an effect only achieved when high doses of free ampicillin were used.

\section{CONCLUDING REMARKS AND PERSPECTIVES.}

Up to now, no antibiotic therapy has been reported to eradicate most intracellular bacteria such us Brucella or Mycobaterium. Moreover, a prolonged exposure to combined antibiotics are required to reduce the disease relapses down to 5$15 \%$. In this sense, DDS have an important role in the management of intracellular infections. As stated above, formulation of antimicrobials in DDS can reduce the side-effects of these drugs and increase patient compliance, thus saving money on health care delivery (115). In this setting, the potential use of DDS loaded with antibiotics may be one of the most relevant therapeutic advances in the treatment of intracellular bacterial diseases in recent years. Liposomes have shown good potential in improving the efficacy and tolerability of antibiotics of current use, however, problems concerning their stability during storage and administration require rigorous attention. On the other hand, polymeric particles, mainly nanoparticles, have emerged more recently as attractive carriers for the delivery of drugs to infected cells. Synthetic biodegradable and biocompatible polymers have been shown to be effective for encapsulating a great variety of antibiotics. Moreover, these polymeric particles strongly enhance phagocytosis and are suitable for intracellular delivery of antibacterial agents. Therefore, it is likely that soon after confirmation of the safety properties of these antibiotic-loaded DDS pilot studies in humans could be initiated.

\section{ACKNOWLEDGMENTS}

This work was supported by the Ministerio de Ciencia e Innovación, Spain (NAN2004-09159-C04-03). Fellowships from the Asociación de Amigos de la Universidad de Navarra, Gobierno de Navarra and Ministerio de Ciencia e Innovación to E. Imbuluzqueta are also gratefully acknowledged.

\section{REFERENCES}

1. J. D. Butts: Intracellular concentrations of antibacterial agents and related clinical implications. Clin Pharmacokinet 27(1), 6384 (1994)

2. A. Alonso and F. Garcia-del Portillo: Hijacking of eukaryotic functions by intracellular bacterial pathogens. Int Microbiol 7(3), 181-191 (2004)

3. M. Desjardins: Biogenesis of phagolysosomes: the kiss and run hypothesis. Trends in Cell Biology 5(5), 183-186 (1995)

4. W. Beron, C. Alvarez-Dominguez, L. Mayorga and P. D. Stahl: Membrane trafficking along the phagocytic pathway. Trends in Cell Biology 5(3), 100-104 (1995)

5. M. Desjardins, L. A. Huber, R. G. Parton and G. Griffiths: Biogenesis of phagolysosomes proceeds through a sequential series of interactions with the endocytic apparatus. J. Cell Biol. 124(5), 677-688 (1994)

6.T. Suzuki and C. Sasakawa: Molecular basis of the intracellular spreading of Shigella. Infect Immun 69(10), 5959-5966 (2001) 


\section{DDS against intracellular infections}

7. D. A. Portnoy, V. Auerbuch and I. J. Glomski: The cell biology of Listeria monocytogenes infection: the intersection of bacterial pathogenesis and cell-mediated immunity. J Cell Biol 158(3), 409-414 (2002)

8. S. Sturgill-Koszycki and M. S. Swanson: Legionella pneumophila replication vacuoles mature into acidic, endocytic organelles. J Exp Med 192(9), 1261-1272 (2000)

9. R. A. Heinzen, M. A. Scidmore, D. D. Rockey and T. Hackstadt: Differential interaction with endocytic and exocytic pathways distinguish parasitophorous vacuoles of Coxiella burnetii and Chlamydia trachomatis. Infect Immun 64(3), 796-809 (1996)

10. J. Pieters and J. Gatfield: Hijacking the host: survival of pathogenic mycobacteria inside macrophages. Trends Microbiol $10(3), 142-146(2002)$

11. D. J. Hackam, O. D. Rotstein, W. Zhang, S. Gruenheid, P. Gros and S. Grinstein: Host resistance to intracellular infection: mutation of natural resistance-associated macrophage protein 1 (Nramp1) impairs phagosomal acidification. $J$ Exp Med 188(2), $351-364(1998)$

12. S. Sturgill-Koszycki, U. E. Schaible and D. G. Russell: Mycobacterium-containing phagosomes are accessible to early endosomes and reflect a transitional state in normal phagosome biogenesis. EMBO J 15(24), 6960-6968 (1996)

13. O. Steele-Mortimer: The Salmonella-containing vacuole: moving with the times. Curr Opin Microbiol 11(1), 38-45 (2008)

14. R. E. Barnewall, Y. Rikihisa and E. H. Lee: Ehrlichia chaffeensis inclusions are early endosomes which selectively accumulate transferrin receptor. Infect Immun 65(4), 1455-1461 (1997)

15. J. Celli: Surviving inside a macrophage: the many ways of Brucella. Res Microbiol 157(2), 93-98 (2006)

16. P. A. Kyme, A. Haas, M. Schaller, A. Peschel, J. Iredell and V. A. Kempf: Unusual trafficking pattern of Bartonella henselae -containing vacuoles in macrophages and endothelial cells. Cell Microbiol 7(7), 1019-1034 (2005)

17. A. Luhrmann, K. Streker, A. Schuttfort, J. J. Daniels and A. Haas: Afipia felis induces uptake by macrophages directly into a nonendocytic compartment. Proc Natl Acad Sci U S A 98(13), 7271-7276 (2001)

18. E. Ghigo, C. Capo, C. H. Tung, D. Raoult, J. P. Gorvel and J. L. Mege: Coxiella burnetii survival in THP-1 monocytes involves the impairment of phagosome maturation: IFN-gamma mediates its restoration and bacterial killing. J Immunol 169(8), 4488-4495 (2002)

19. C. Pujol and J. B. Bliska: The ability to replicate in macrophages is conserved between Yersinia pestis and Yersinia pseudotuberculosis. Infect Immun 71(10), 5892-5899 (2003)

20. H. Tsukano, F. Kura, S. Inoue, S. Sato, H. Izumiya, T. Yasuda and H. Watanabe: Yersinia pseudotuberculosis blocks the phagosomal acidification of B10.A mouse macrophages through the inhibition of vacuolar $\mathrm{H}(+)$-ATPase activity. Microb Pathog 27(4), 253-263 (1999)

21. D. L. Clemens, B. Y. Lee and M. A. Horwitz: Virulent and avirulent strains of Francisella tularensis prevent acidification and maturation of their phagosomes and escape into the cytoplasm in human macrophages. Infect Immun 72(6), 3204-3217 (2004)

22. M. Kubica, K. Guzik, J. Koziel, M. Zarebski, W. Richter, B. Gajkowska, A. Golda, A. Maciag-Gudowska, K. Brix, L. Shaw, T. Foster and J. Potempa: A potential new pathway for Staphylococcus aureus dissemination: the silent survival of S. aureus phagocytosed by human monocyte-derived macrophages. PLoS One 3(1), e1409 (2008)

23. M. Bonazzi and P. Cossart: Bacterial entry into cells: a role for the endocytic machinery. FEBS Lett 580(12), 2962-2967 (2006)

24. M. B. Goldberg and J. A. Theriot: Shigella flexneri surface protein IcsA is sufficient to direct actin-based motility. Proc Natl Acad Sci U S A 92(14), 6572-6576 (1995)

25. S. Hashim, K. Mukherjee, M. Raje, S. K. Basu and A. Mukhopadhyay: Live Salmonella Modulate Expression of Rab Proteins to Persist in a Specialized Compartment and Escape Transport to Lysosomes. J. Biol. Chem. 275(21), 16281-16288 (2000)

26. G. Ferrari, H. Langen, M. Naito and J. Pieters: A coat protein on phagosomes involved in the intracellular survival of mycobacteria. Cell 97(4), 435-447 (1999) 


\section{DDS against intracellular infections}

27. M. A. Scidmore, E. R. Fischer and T. Hackstadt: Restricted fusion of Chlamydia trachomatis vesicles with endocytic compartments during the initial stages of infection. Infect Immun 71(2), 973-984 (2003)

28. C. van Ooij, L. Kalman, I. van, M. Nishijima, K. Hanada, K. Mostov and J. N. Engel: Host cell-derived sphingolipids are required for the intracellular growth of Chlamydia trachomatis. Cell Microbiol 2(6), 627-637 (2000)

29. A. D. Joshi, S. Sturgill-Koszycki and M. S. Swanson: Evidence that Dot-dependent and -independent factors isolate the Legionella pneumophila phagosome from the endocytic network in mouse macrophages. Cell Microbiol 3(2), 99-114 (2001)

30. C. R. Roy and L. G. Tilney: The road less traveled: transport of Legionella to the endoplasmic reticulum. J Cell Biol 158(3), 415-419 (2002)

31. B. Arellano-Reynoso, N. Lapaque, S. Salcedo, G. Briones, A. E. Ciocchini, R. Ugalde, E. Moreno, I. Moriyon and J.-P. Gorvel: Cyclic [beta]-1,2-glucan is a brucella virulence factor required for intracellular survival. Nat Immunol 6(6), 618-625 (2005)

32. T. Starr, T. W. Ng, T. D. Wehrly, L. A. Knodler and J. Celli: Brucella Intracellular Replication Requires Trafficking Through the Late Endosomal/Lysosomal Compartment. Traffic 9(5), 678-694 (2008)

33. F. Van Bambeke, J. M. Michot and P. M. Tulkens: Antibiotic efflux pumps in eukaryotic cells: occurrence and impact on antibiotic cellular pharmacokinetics, pharmacodynamics and toxicodynamics. J Antimicrob Chemother 51(5), 1067-1077 (2003)

34. M. Barcia-Macay, C. Seral, M.-P. Mingeot-Leclercq, P. M. Tulkens and F. Van Bambeke: Pharmacodynamic Evaluation of the Intracellular Activities of Antibiotics against Staphylococcus aureus in a Model of THP-1 Macrophages. Antimicrob. Agents Chemother. 50(3), 841-851 (2006)

35. S. Carryn, F. Van Bambeke, M. P. Mingeot-Leclercq and P. M. Tulkens: Comparative intracellular (THP-1 macrophage) and extracellular activities of beta-lactams, azithromycin, gentamicin, and fluoroquinolones against Listeria monocytogenes at clinically relevant concentrations. Antimicrob Agents Chemother 46(7), 2095-2103 (2002)

36. M.-P. Mingeot-Leclercq, Y. Glupczynski and P. M. Tulkens: Aminoglycosides: Activity and Resistance. Antimicrob. Agents Chemother. 43(4), 727-737 (1999)

37. M.-P. Mingeot-Leclercq and P. M. Tulkens: Aminoglycosides: Nephrotoxicity. Antimicrob. Agents Chemother. 43(5), 1003$1012(1999)$

38. C. Seral, F. Van Bambeke and P. M. Tulkens: Quantitative analysis of gentamicin, azithromycin, telithromycin, ciprofloxacin, moxifloxacin, and oritavancin (LY333328) activities against intracellular Staphylococcus aureus in mouse J774 macrophages. Antimicrob Agents Chemother 47(7), 2283-2292 (2003)

39. S. Domingo, I. Gastearena, R. Diaz and C. Gamazo: Significance of environmental conditions ( $\mathrm{pH}$ and serum) on the in vitro potency of azithromycin against Brucella melitensis. J Chemother 7 Suppl 4, 29-31 (1995)

40. M. B. Carlier, B. Scorneaux, A. Zenebergh, J. F. Desnottes and P. M. Tulkens: Cellular uptake, localization and activity of fluoroquinolones in uninfected and infected macrophages. J Antimicrob Chemother 26 Suppl B, 27-39 (1990)

41. A. L. Baltch, L. H. Bopp, R. P. Smith, P. B. Michelsen and W. J. Ritz: Antibacterial activities of gemifloxacin, levofloxacin, gatifloxacin, moxifloxacin and erythromycin against intracellular Legionella pneumophila and Legionella micdadei in human monocytes. J Antimicrob Chemother 56(1), 104-109 (2005)

42. H. A. Nguyen, J. Grellet, D. Paillard, V. Dubois, C. Quentin and M. C. Saux: Factors influencing the intracellular activity of fluoroquinolones: a study using levofloxacin in a Staphylococcus aureus THP-1 monocyte model. J Antimicrob Chemother 57(5), 883-890 (2006)

43. P. Gilbert, P. J. Collier and M. R. Brown: Influence of growth rate on susceptibility to antimicrobial agents: biofilms, cell cycle, dormancy, and stringent response. Antimicrob Agents Chemother 34(10), 1865-1868 (1990)

44. A. M. Cuffini, V. Tullio, N. Mandras, J. Roana, G. Banche and N. A. Carlone: The leading role of antimicrobial agents in modulating the binomial host-microorganism Curr. Med. Chem.-Anti-Infective Agents 3, 1-13 (2004) 


\section{DDS against intracellular infections}

45. D. A. Drevets, B. P. Canono, P. J. Leenen and P. A. Campbell: Gentamicin kills intracellular Listeria monocytogenes. Infect Immun 62(6), 2222-2228 (1994)

46. M. T. Labro: Interference of antibacterial agents with phagocyte functions: immunomodulation or "immuno-fairy tales"? Clin Microbiol Rev 13(4), 615-650 (2000)

47. P. J. van den Broek: Antimicrobial drugs, microorganisms, and phagocytes. Rev Infect Dis 11(2), $213-245$ (1989)

48. S. Prior, B. Gander, N. Blarer, H. P. Merkle, M. L. Subira, J. M. Irache and C. Gamazo: In vitro phagocytosis and monocytemacrophage activation with poly(lactide) and poly(lactide-co-glycolide) microspheres. Eur J Pharm Sci 15(2), 197-207 (2002)

49. G. Gregoriadis: The carrier potential of liposomes in biology and medicine (first of two parts). N Engl J Med 295(13), 704$710(1976)$

50. F. Ahsan, I. P. Rivas, M. A. Khan and A. I. Torres Suarez: Targeting to macrophages: role of physicochemical properties of particulate carriers--liposomes and microspheres--on the phagocytosis by macrophages. J Control Release 79(1-3), 29-40 (2002)

51. P. Couvreur, E. Fattal and A. Andremont: Liposomes and nanoparticles in the treatment of intracellular bacterial infections. Pharm Res 8(9), 1079-1786 (1991)

52. C. Kirby and G. Gregoriadis: Plasma-induced release of solutes from small unilamellar liposomes is associated with pore formation in the bilayers. Biochem J 199(1), 251-254 (1981)

53. G. Scherphof, F. Roerdink, M. Waite and J. Parks: Disintegration of phosphatidylcholine liposomes in plasma as a result of interaction with high-density lipoproteins. Biochim Biophys Acta 542(2), 296-307 (1978)

54. T. M. Allen, G. A. Austin, A. Chonn, L. Lin and K. C. Lee: Uptake of liposomes by cultured mouse bone marrow macrophages: influence of liposome composition and size. Biochim Biophys Acta 1061(1), 56-64 (1991)

55. D. L. Daleke, K. Hong and D. Papahadjopoulos: Endocytosis of liposomes by macrophages: binding, acidification and leakage of liposomes monitored by a new fluorescence assay. Biochim Biophys Acta 1024(2), 352-366 (1990)

56. J. Dijkstra, M. van Galen and G. Scherphof: Influence of liposome charge on the association of liposomes with Kupffer cells in vitro. Effects of divalent cations and competition with latex particles. Biochim Biophys Acta 813(2), 287-297 (1985)

57. A. I. Vitas, R. Diaz and C. Gamazo: Effect of composition and method of preparation of liposomes on their stability and interaction with murine monocytes infected with Brucella abortus. Antimicrob Agents Chemother 40(1), 146-151 (1996)

58. C. J. Chu, J. Dijkstra, M. Z. Lai, K. Hong and F. C. Szoka: Efficiency of cytoplasmic delivery by pH-sensitive liposomes to cells in culture. Pharm Res 7(8), 824-834 (1990)

59. V. A. Slepushkin, S. Simoes, P. Dazin, M. S. Newman, L. S. Guo, M. C. Pedroso de Lima and N. Duzgunes: Sterically stabilized $\mathrm{pH}$-sensitive liposomes. Intracellular delivery of aqueous contents and prolonged circulation in vivo. J Biol Chem 272(4), 2382-2388 (1997)

60. K. D. Lee, Y. K. Oh, D. A. Portnoy and J. A. Swanson: Delivery of macromolecules into cytosol using liposomes containing hemolysin from Listeria monocytogenes. J Biol Chem 271(13), 7249-7252 (1996)

61. Salem, II, D. L. Flasher and N. Duzgunes: Liposome-encapsulated antibiotics. Methods Enzymol 391, 261-291 (2005)

62. C. O. Onyeji, C. H. Nightingale, D. P. Nicolau and R. Quintiliani: Efficacies of liposome-encapsulated clarithromycin and ofloxacin against Mycobacterium avium-M. intracellulare complex in human macrophages. Antimicrob Agents Chemother 38(3), 523-527 (1994)

63. C. O. Onyeji, C. H. Nightingale, D. P. Nicolau and R. Quintiliani: Activities of liposome-encapsulated azithromycin and rifabutin compared with that of clarithromycin against Mycobacterium avium-intracellulare complex in human macrophages. Int J Antimicrob Agents 4(4), 281-289 (1994)

64. Y. K. Oh, D. E. Nix and R. M. Straubinger: Formulation and efficacy of liposome-encapsulated antibiotics for therapy of intracellular Mycobacterium avium infection. Antimicrob Agents Chemother 39(9), 2104-2111 (1995) 


\section{DDS against intracellular infections}

65. N. Duzgunes, D. Flasher, M. V. Reddy, J. Luna-Herrera and P. R. Gangadharam: Treatment of intracellular Mycobacterium avium complex infection by free and liposome-encapsulated sparfloxacin. Antimicrob Agents Chemother 40(11), 2618-2621 (1996)

66. S. Majumdar, D. Flasher, D. S. Friend, P. Nassos, D. Yajko, W. K. Hadley and N. Duzgunes: Efficacies of liposomeencapsulated streptomycin and ciprofloxacin against Mycobacterium avium-M. intracellulare complex infections in human peripheral blood monocyte/macrophages. Antimicrob Agents Chemother 36(12), 2808-2815 (1992)

67. R. Gomez-Flores, R. Hsia, R. Tamez-Guerra and R. T. Mehta: Enhanced intramacrophage activity of resorcinomycin A against Mycobacterium avium-Mycobacterium intracellulare complex after liposome encapsulation. Antimicrob Agents Chemother 40(11), 2545-2549 (1996)

68. P. Lutwyche, C. Cordeiro, D. J. Wiseman, M. St-Louis, M. Uh, M. J. Hope, M. S. Webb and B. B. Finlay: Intracellular delivery and antibacterial activity of gentamicin encapsulated in $\mathrm{pH}$-sensitive liposomes. Antimicrob Agents Chemother 42(10), 2511-2520 (1998)

69. P. F. Bonventre and G. Gregoriandis: Killing of intraphagocytic Staphylococcus aureus by dihydrostreptomycin entrapped within liposomes. Antimicrob Agents Chemother 13(6), 1049-1051 (1978)

70. J. Roesler, S. Hockertz, B. Vogt and M. L. Lohmann-Matthes: Staphylococci surviving intracellularly in phagocytes from patients suffering from chronic granulomatous disease are killed in vitro by antibiotics encapsulated in liposomes. $J$ Clin Invest 88(4), 1224-1229 (1991)

71. I. A. Bakker-Woudenberg, A. F. Lokerse and F. H. Roerdink: Effect of lipid composition on activity of liposome-entrapped ampicillin against intracellular Listeria monocytogenes. Antimicrob Agents Chemother 32(10), 1560-1564 (1988)

72. M. Ito, E. Ishida, F. Tanabe, N. Mori and S. Shigeta: Inhibitory effect of liposome-encapsulated penicillin G on growth of Listeria monocytogenes in mouse macrophages. Tohoku J Exp Med 150(3), 281-286 (1986)

73. R. C. Mundargi, V. R. Babu, V. Rangaswamy, P. Patel and T. M. Aminabhavi: Nano/micro technologies for delivering macromolecular therapeutics using poly(D,L-lactide-co-glycolide) and its derivatives. J Control Release 125(3), 193-209 (2008)

74. R. A. Jain: The manufacturing techniques of various drug loaded biodegradable poly(lactide-co-glycolide) (PLGA) devices. Biomaterials 21(23), 2475-2490 (2000)

75. F. Tewers, F. Boury and J. P. Benoit: Biodegradable Microspheres: Advances in Production Technology. In: Microencapsulation: Methods and Industrial Applications. Ed S. Benita. Marcel Dekker, New York (2006)

76. N. Wakiyama, K. Juni and M. Nakano: Preparation and evaluation in vitro and in vivo of polylactic acid microspheres containing dibucaine. Chem Pharm Bull (Tokyo) 30(10), 3719-3727 (1982)

77. N. Manson, C. Thies and T. J. Cicero: In vivo and in vitro evaluation of a microencapsulated narcotic antagonist. J. Pharm. Sci. 65, 51-59(1976)

78. M. Kanke, E. Morlier, R. Geissler, D. Powell, A. Kaplan and P. P. DeLuca: Interaction of microspheres with blood constituents II: Uptake of biodegradable particles by macrophages. J Parenter Sci Technol 40(4), 114-118 (1986)

79. P. D. A. Hart and M. R. Young: Interference with normal phagosome-lysosome fusion in macrophages, using ingested yeast cells and suramin. Nature 256(5512), 47-49 (1975)

80. P. L. Small, L. Ramakrishnan and S. Falkow: Remodeling schemes of intracellular pathogens. Science 263(5147), 637-639 (1994)

81. Y. K. Oh and J. A. Swanson: Different fates of phagocytosed particles after delivery into macrophage lysosomes. J Cell Biol 132(4), 585-593 (1996)

82. R. S. Raghuvanshi, Y. K. Katare, K. Lalwani, M. M. Ali, O. Singh and A. K. Panda: Improved immune response from biodegradable polymer particles entrapping tetanus toxoid by use of different immunization protocol and adjuvants. Int $J$ Pharm 245(1-2), 109-121 (2002)

83. C. de Chastellier and L. Thilo: Phagosome maturation and fusion with lysosomes in relation to surface property and size of the phagocytic particle. Eur J Cell Biol 74(1), 49-62 (1997) 


\section{DDS against intracellular infections}

84. M. Koval, K. Preiter, C. Adles, P. D. Stahl and T. H. Steinberg: Size of IgG-opsonized particles determines macrophage response during internalization. Exp Cell Res 242(1), 265-273 (1998)

85. M. C. Lecaroz, M. J. Blanco-Prieto, M. A. Campanero, H. Salman and C. Gamazo: Poly(D,L-lactide-coglycolide) particles containing gentamicin: pharmacokinetics and pharmacodynamics in Brucella melitensis-infected mice. Antimicrob Agents Chemother 51(4), 1185-1190 (2007)

86. M. Murillo, C. Gamazo, M. Goni, J. Irache and M. Blanco-Prieto: Development of microparticles prepared by spray-drying as a vaccine delivery system against brucellosis. Int J Pharm 242(1-2), 341-344 (2002)

87. P. Artursson, E. Arro, P. Edman, J. L. Ericsson and I. Sjoholm: Biodegradable microspheres. V: Stimulation of macrophages with microparticles made of various polysaccharides. J Pharm Sci 76(2), 127-33 (1987)

88. B. Stringer and L. Kobzik: Alveolar macrophage uptake of the environmental particulate titanium dioxide: role of surfactant components. Am J Respir Cell Mol Biol 14(2), 155-160 (1996)

89. S. Prior, B. Gander, C. Lecaroz, J. M. Irache and C. Gamazo: Gentamicin-loaded microspheres for reducing the intracellular Brucella abortus load in infected monocytes. J Antimicrob Chemother 53(6), 981-988 (2004)

90. R. Sharma, P. Muttil, A. B. Yadav, S. K. Rath, V. K. Bajpai, U. Mani and A. Misra: Uptake of inhalable microparticles affects defence responses of macrophages infected with Mycobacterium tuberculosis H37Ra. J Antimicrob Chemother 59(3), 499-506 (2007)

91. C. Lecaroz, M. J. Blanco-Prieto, M. A. Burrell and C. Gamazo: Intracellular killing of Brucella melitensis in human macrophages with microsphere-encapsulated gentamicin. J Antimicrob Chemother 58(3), 549-556 (2006)

92. C. Lecaroz, C. Gamazo and M. J. Blanco-Prieto: Nanocarriers with gentamicin to treat intracellular pathogens. J Nanosci Nanotechnol 6(9-10), 3296-302 (2006)

93. M. N. Seleem, N. Jain, N. Pothayee, A. Ranjan, J. S. Riffle and N. Sriranganathan: Targeting Brucella melitensis with polymeric nanoparticles containing streptomycin and doxycycline. FEMS Microbiol Lett 294(1), 24-31 (2009)

94. H. Pinto-Alphandary, O. Balland, M. Laurent, A. Andremont, F. Puisieux and P. Couvreur: Intracellular visualization of ampicillin-loaded nanoparticles in peritoneal macrophages infected in vitro with Salmonella typhimurium. Pharm Res 11(1), 38$46(1994)$

95. O. Balland, H. Pinto-Alphandary, A. Viron, E. Puvion, A. Andremont and P. Couvreur: Intracellular distribution of ampicillin in murine macrophages infected with Salmonella typhimurium and treated with $(3 \mathrm{H})$ ampicillin-loaded nanoparticles. $J$ Antimicrob Chemother 37(1), 105-115 (1996)

96. F. Forestier, P. Gerrier, C. Chaumard, A. M. Quero, P. Couvreur and C. Labarre: Effect of nanoparticle-bound ampicillin on the survival of Listeria monocytogenes in mouse peritoneal macrophages. J Antimicrob Chemother 30(2), 173-179 (1992)

97. S. Labana, R. Pandey, S. Sharma and G. K. Khuller: Chemotherapeutic activity against murine tuberculosis of once weekly administered drugs (isoniazid and rifampicin) encapsulated in liposomes. Int J Antimicrob Agents 20(4), 301-304 (2002)

98. R. Pandey, A. Sharma, A. Zahoor, S. Sharma, G. K. Khuller and B. Prasad: Poly (DL-lactide-co-glycolide) nanoparticlebased inhalable sustained drug delivery system for experimental tuberculosis. J Antimicrob Chemother 52(6), 981-986 (2003)

99. S. Suarez, P. O'Hara, M. Kazantseva, C. E. Newcomer, R. Hopfer, D. N. McMurray and A. J. Hickey: Respirable PLGA microspheres containing rifampicin for the treatment of tuberculosis: screening in an infectious disease model. Pharm Res 18(9), $1315-1319(2001)$

100. S. Suarez, P. O'Hara, M. Kazantseva, C. E. Newcomer, R. Hopfer, D. N. McMurray and A. J. Hickey: Airways delivery of rifampicin microparticles for the treatment of tuberculosis. J Antimicrob Chemother 48(3), 431-434 (2001)

101. Q. Ul-Ain, S. Sharma and G. K. Khuller: Chemotherapeutic potential of orally administered poly(lactide-co-glycolide) microparticles containing isoniazid, rifampin, and pyrazinamide against experimental tuberculosis. Antimicrob Agents Chemother 47(9), 3005-3007 (2003) 


\section{DDS against intracellular infections}

102. R. Pandey, A. Zahoor, S. Sharma and G. K. Khuller: Nanoparticle encapsulated antitubercular drugs as a potential oral drug delivery system against murine tuberculosis. Tuberculosis (Edinb) 83(6), 373-378 (2003)

103. J. Ariza, F. Gudiol, R. Pallares, P. F. Viladrich, G. Rufi, J. Corredoira and M. R. Miravitlles: Treatment of human brucellosis with doxycycline plus rifampin or doxycycline plus streptomycin. A randomized, double-blind study. Ann Intern Med 117(1), 25-30 (1992)

104. J. Solera, A. Espinosa, E. Martinez-Alfaro, L. Sanchez, P. Geijo, E. Navarro, J. Escribano and J. A. Fernandez: Treatment of human brucellosis with doxycycline and gentamicin. Antimicrob Agents Chemother 41(1), 80-84 (1997)

105. A. I. Vitas, R. Diaz and C. Gamazo: Protective effect of liposomal gentamicin against systemic acute murine brucellosis. Chemotherapy 43(3), 204-210 (1997)

106. E. Fattal, M. Youssef, P. Couvreur and A. Andremont: Treatment of experimental salmonellosis in mice with ampicillinbound nanoparticles. Antimicrob Agents Chemother 33(9), 1540-1543 (1989)

107. M. E. Page-Clisson, H. Pinto-Alphandary, E. Chachaty, P. Couvreur and A. Andremont: Drug targeting by polyalkylcyanoacrylate nanoparticles is not efficient against persistent Salmonella. Pharm Res 15(4), 544-549 (1998)

108. E. Fattal, J. Rojas, M. Youssef, P. Couvreur and A. Andremont: Liposome-entrapped ampicillin in the treatment of experimental murine listeriosis and salmonellosis. Antimicrob Agents Chemother 35(4), 770-772 (1991)

109. T. Tadakuma, N. Ikewaki, T. Yasuda, M. Tsutsumi, S. Saito and K. Saito: Treatment of experimental salmonellosis in mice with streptomycin entrapped in liposomes. Antimicrob Agents Chemother 28(1), 28-32 (1985)

110. C. E. Swenson, K. A. Stewart, J. L. Hammett, W. E. Fitzsimmons and R. S. Ginsberg: Pharmacokinetics and in vivo activity of liposome-encapsulated gentamicin. Antimicrob. Agents Chemother. 34(2), 235-240 (1990)

111. J. Fierer, L. Hatlen, J. P. Lin, D. Estrella, P. Mihalko and A. Yau-Young: Successful treatment using gentamicin liposomes of Salmonella dublin infections in mice. Antimicrob Agents Chemother 34(2), 343-348 (1990)

112. C. Cordeiro, D. J. Wiseman, P. Lutwyche, M. Uh, J. C. Evans, B. B. Finlay, and M. S. Webb: Antibacterial efficacy of gentamicin encapsulated in $\mathrm{pH}$-sensitive liposomes against an in vivo Salmonella enterica serovar Typhimurium intracellular infection model. Antimicrob Agents Chemother 44(3), 533-539 (2000)

113. H. Hof: Listeriosis: therapeutic options. FEMS Immunol Med Microbiol 35(3), 203-205 (2003)

114. M. Youssef, E. Fattal, M. J. Alonso, L. Roblot-Treupel, J. Sauzieres, C. Tancrede, A. Omnes, P. Couvreur and A. Andremont: Effectiveness of nanoparticle-bound ampicillin in the treatment of Listeria monocytogenes infection in athymic nude mice. Antimicrob Agents Chemother 32(8), 1204-1207 (1988)

115. G. A. Hughes: Nanostructure-mediated drug delivery. Nanomedicine: Nanotechnology, Biology and Medicine 1(1), 22-30

Abbreviations: DDS: drug delivery systems, ER: endoplasmic reticulum, MPS: mononuclear phagocytic system, SUV: small unilamellar vesicles, LUV: large unilamellar vesicles, SPLV: small plurilamellar vesicles, DOPE: dioleyl phosphatidylethanolamine, MAC: Mycobacterium avium complex, CFU: colony forming units, ROI: reactive oxygen intermediates, PLA: poly(lactic acid), PLGA: poly(lactic co-glycolic acid), PEO-b-PtBA: poly(ethylene oxide)-b-poly(tert-butyl acrylate), PIHCA: polyisohexylcyanoacrylate

Key words: Intracellular Bacteria, Antibiotic Treatment, Drug Delivery System, Microparticle, Nanoparticle, Liposome, Review

Sent correspondence to: Maria J. Blanco-Prieto, Department of Pharmacy and Pharmaceutical Technology, University of Navarra, Irunlarrea 1, 31080 Pamplona, Spain, Tel: 34 948425600, Fax: 34 948425649, E-mail: mjblanco@unav.es

Table 1. Main intracellular bacteria that survive within phagocytic cells

\begin{tabular}{|l|l|l|l|l|}
\hline Organism & Type of parasite & Subcellular localization & pH & Ref. \\
\hline Shigella spp. & Facultative & Cytosol & $\sim 6.5$ & $(6)$ \\
\hline Listeria monocytogenes & Facultative & Cytosol & $\sim 6.5$ & $(7)$ \\
\hline Legionella pneumophila & Facultative & Phagolysosomes, endoplasmic reticulum & 5.6 & $(8)$ \\
\hline Clamydia spp. & Strict & Inclusions (nonacidified vacuoles) & $>6.0$ & $(9)$ \\
\hline
\end{tabular}




\begin{tabular}{|l|l|l|l|l|}
\hline $\begin{array}{l}\text { Mycobacterium } \\
\text { tuberculosis }\end{array}$ & Facultative & Early endosomes & ${ }^{1}$ ND & $(10)$ \\
\hline Mycobacterium bovis & Facultative & Early endosomes & 5.5 & $(11)$ \\
\hline Mycobacterium avium & Facultative & Early endosomes & $(5.6-6.3)$ & $(12)$ \\
\hline Salmonella spp. & Facultative & Modified phagosome (Salmonella-containing vacuole) & $<4.5$ & $(13)$ \\
\hline Ehrlichia chaffeensis & Strict & Early endosome & ND & $(14)$ \\
\hline Brucella spp. & Facultative & Endoplamic reticulum & ND & $(15)$ \\
\hline Bartonella hesenlae & Facultative & Specialized nonendocytic membrane-bound vacuole & $(16)$ \\
\hline Afipia felis & Facultative & Unfused vacuoles & ND & $(17)$ \\
\hline Coxiella burnetii & Strict & Phagolysosome & $\sim 5$ & $(18)$ \\
\hline Yersinia pestis & Facultative & Phagolysosomes & ND & $(19)$ \\
\hline $\begin{array}{l}\text { Yersinia } \\
\text { pseudotuberculosis }\end{array}$ & Facultative & Phagosomes & 6.0 & $(20)$ \\
\hline Francisella tularensis & Facultative & Phagosomes & 6.7 & $(21)$ \\
\hline Staphylococcus. aureus & Facultative & Phagolysosome & ND & $(22)$ \\
\hline
\end{tabular}

Abbreviations: ${ }^{1} \mathrm{ND}=$ Not determined.

Figure 1. Bacterial phagocytosis and phagolysosome biogenesis. In the endocytic pathway, internalized molecules are delivered to early endosomes, following recycling of part of this network of tubules and cisternae (containing receptors) back to the plasma membrane, whereas other components of the endosome are transported to late endosomes and lysosomes for degradation. On the other hand, phagocytosis and phagosome maturation are coordinated with the endocytic pathway through sequential activities of molecules that regulate the cytoskeleton and direct fusion and other contact events between components of the phagocytic and the endocytic pathways. Professional phagocytes enclose the adsorbed microorganism into membrane-bound organelles called phagosomes. Following internalization, this process leads to phagosome maduration as a sequential process involving fusion with components of the natural endocytic pathway (early endosomes, late endosomes and lysosomes), ultimately forming the phagolysosome. In the phagolysosome the ingested materials are finally degraded. Maturation in both endocytic and phagocytic pathways are also accompanied by changes in the vesicular markers content. Changes in the phospholipid composition are also observed during phagosome maturation. While early phagosomes are enriched with phosphatidylcholine, late phagosomes are preferentially enriched with sphingomyelin.

Figure 2. Different intracellular fate of conventional and $\mathrm{pH}$-sensitive liposomes. Conventional liposomes are delivered to lysosomes (LYS) and degraded, releasing the contained drug. $\mathrm{pH}$-sensitive liposomes also traffic through early and late endosomes (EE and LE) to lysosomes, where they are unstable. At the acidic pH of lysosomes, these liposomes are able to destabilize the lysosomeal membrane or fuse to it, releasing their drug content into the cell cytoplasm.

Figure 3. Intracellular trafficking of polymeric particles. The polymeric particle surface needs to stimulate binding to the cellular membrane to initiate phagocytosis. Two principal mechanisms have been discussed to explain the internalization of particles into cells: the zipper-like and the trigger-like mechanisms. In the zipper mechanism, pseudopods go forward to engulf particles, probably due to their high affinity toward the cell membrane, resulting in the formation of tightly apposed phagosomes. These events occur in the case of cationic or hydrophobic particles. In contrast, hydrophilic or anionic particles are phagocytosed without formation of pseudopods and end up in loosely apposed phagosomes. As a trigger mechanism, the particle would be ingested in a sinking-in-to-the-cell-like manner, in loose contact with the phagosomal membrane. Phagocytosis leads to the intracellular entrapment of particles in phagosomes, which mature under the influence of acidification, resulting from their fusion with lysosomes (LYS). The process of phagosome maturation has been shown to relate to the degree of apposition of the phagosomal membrane to the entrapped particle. -Hydrophobic particles tightly fitted in phagosomes inhibit maturation by resisting rapid fusion with lysosomes, because of the ionic interactions with the negatively charged phagosomal membrane. These particles are therefore found in relatively neutral microenvironments (6.0-6.8) and in tight apposition to the phagosomal membrane. -Hydrophilic particles, entrapped loosely in the phagosomes, are subjected to rapid fusion with lysosomes. These particles are then found in more acidic microenvironments typical of matured phagosomes ( $\mathrm{pH} 4.6$ to 5.1). Abbreviations: EE = early endosome; $\mathrm{LE}=$ late endosome.

Figure 4. Transmission electron micrographs of THP-1 human monocyte engulfing both gentamicin-containing microparticles (MP) and Brucella melitensis cells (BM). White microparticles and dark brucellae can be seen throughout the monocyte. Microparticles ranged in size around $1.0 \mu \mathrm{m}$ in diameter and appeared as discrete membrane-bound particles. Gentamicin was detected (immunogold dots) inside microparticles and also inside Brucella's compartment. (Original magnification x 10.000). 